and the general evidence of a protracted dissipation, which he admitted.

He could scarcely speak in an audible tone, and stated that the voice commenced to weaken eighteen months previous.

He had been compelled to relinquish his company, as the puerile voice rendered proper decorum among his men an impossibility, for they could not hear his commands.

The laryngoscope revealed a moderately congested pharyngeal and laryngeal mucous membrane, no erosions, hypertrophies, nor inter-arytenoid thickening.

Upon attempted phonation the vocal bands were incompletely approximated, and much phonetic leakage occurred.

The treatment instituted consisted in enforced abstinence from all alcoholic stimulants, the internal administration of thirty minims of tincture of nux vomica thrice daily.

Sprays of an ethereal solution of iodoform $2.3^{\circ}$ gms. to $21.00 \mathrm{gms}$., as well as ammonium chloride

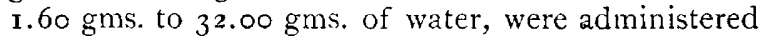
on alternate days, morning and evening.

Improvement followed, and the patient said that his own children were frightened at his voice on re. turning after four weeks' absence from home.

In six weeks the voice was completely restored, and he was discharged from medical treatment and rejoined his company.

I learn that he has been more temperate, and that his voice is still good two years after treatment.

CASE II. M. C., aged $\mathbf{3 2}$, white, to whom I was called on the morning of March 30 , I 884, was my second case.

The messenger stated that the patient was experiencing suffocative attacks, and desired my aid immediately.

On arrival I found my patient in a state of nervous excitement. He said in a whisper, devoid of vocal sound, that he had habitually drank alcoholics to excess; but having recently indulged more freely than usual, developed aphonia, insomnia and nervousness.

A laryngoscopic examination showed a normal mucous membrane-no hyperæmia, inflammation or ulceration. The vocal bands, however, failed to approximate during phonation, and on inspiration the same bands evinced indisposition to proper abduction.

A careful physical exploration of the thoracic organs demonstrated the absence of all disease other than slight cardiac hypertrophy, and his intellect, hearing and vision were normal. He had never had syphilis, rheumatism, or any laryngeal inflammation.

The patient said that on a previous occasion he had lost his voice for ten days, during an exacerbation of alcoholism.

My treatment in this case comprised abstinence from alcohol, the administration of a purge of calomel and rhubarb, followed by nervous sedatives and hypnotics. I pencilled the larynx with a pigment of zinc chloride, endeavoring to arouse the torpid muscles.

The patient slept well during the next forty-eight hours, took nourishment, and improved in voice but slowly.
I finally employed faradisation and nux vonica with the best result, as the power of complete phonation was regained in ten or twelve days.

Here are two instances of aphonia existing in patients having normal sight, hearing, and perfect intellectual faculties, patients free from chronic catarrhal laryngitis and the evidence of syphilis or rheumatism.

The removal of the alcohol in one, and the employment of either an excito-motor stimulant or faradisation in the other, resulted in entire restoration of voice in these patients.

The rapid improvement in my cases, and line of treatment, excludes intracranial influence as causing the aphonia.

Hamon ${ }^{1}$ says : "It is totally unnecessary for alcoholic poisoning to be pronounced in order that aphonia may be produced." This statement is in accord with my own observation, and is verified by my cases.

Alcoholic aphonia is, in my opinion, more common than the scanty literature would suggest.

The voice, as Broue ${ }^{2}$ judiciously observes, is the hygrometer of sobriety.

Recalling, then, the many causes involved in the production of paralyses of the lateral crico-arytenoids, and the uncertainty of our knowledge on the entire subject, may I venture to add alcohol to the list of toxics occasioning paralysis of the laryngeal muscles?

At least let alcohol be assigned to the same category as lead, arsenic, antimony and opium, there to remain subject to future scrutiny and additional clinical observation.

\section{TREATMENT OF UTERINE DISPLACEMENTS.}

BY AUGUSTUS P. CLARKE, M.D., CAMBRIDGE, MASS.

Read before the Gynæcological Society of Boston, Nov. I3, 1884 .

Perhaps in no other department of gynæcological practice has a subject been more thoroughly considered than that of the treatment of uterine dispiacements, and although much has been achieved in this direction by the study, still there remains much diversity of opinion in regard to the propriety and method of such treatment. From the earliest times physicians have recognized the discomforts of uterine displacements, and have soughi in some manner to rectify them, and for such relief a great variety of devices have been used. The ancients made use of medicated pessaries, which they distinguished into astringent, opiate, aperient, emollient, etc. The solid pessaries are composed of cork, ivory, wood, gum elastic, sponge, caoutchouc, silver, lead, zinc, copper, glass and other peculiar substances, and often coated or concealed by soft rubber or other protective, as the peculiar symptoms, or as the fancy of the operator might dictate. Indeed it would seem that a physician can hardly be considered a good gynæcologist until he has devised or approved of some special form of a pessary. Without attempting to wade

1 Gaz. d'Hop., Par., x860, xxxiii, p. 222.

2 Hygiene Philosop. d' Artistes Dramatiques, Par., 1836, ii, p. ıog. 
through the history and description of these several singular contrivances, I would state that the main design in theory, at least, in the treatment of uterine flexions is to place the unimpregnated uterus of a woman, otherwise healthy, in such a plane or position in the pelvis that the circulation of that organ shall be unobstructerl. This is the important point in the treatment that should not be lost sight of, if we are to succeed at all in properly selected cases. Another important point in correcting a displacement or prolapse is not to elevate the uterus above the normal health line, for when the elevation is too great, the symptoins of prolapse or displacement will remain unrelieved, and the cervix be liable to become eroded, and a leucorrhoal discharge take place. The enlarged and flabby condition of a retroposed uterus has often been ascribed to subinvolution of that organ, but this condition of hypertrophy is often found in nulliparæ who present themselves for treatment for retroflexion. I can recall cases of retroflexion occurring in nulliparæ when the cervical canal was patulous and when the mucous membrane of the lip.s protruded, presenting the appearance of ectropion.

Such cases are usually attended with a cervical discharge producing more or less excoriation, and often vaginitis. 'The uterus at first, and more especially the fundus, is retroflexed, and if the symptoms remain unrelieved, that organ becomes more and more prolapsed. In this condition the uterus is often extremely sensitive, and the adjacent parts, particularly the ovaries, become the centres of hyperæsthesia. In such a case as this the retroflexion is not the sole cause of this perverted sensibility-but it is owing to the changed relations of the body, fundus and cervix to each other, and the necessary strain upon the uterine ligaments and the production of venous stasis in the parts, by the pressure on the walls of the blood-vessels at the preternatural curve or angle formed by the flexion. In recently produced cases, by sudden violence a good deal of hamorrhage often takes place, but this is not owing to congestion, as is sometimes ascribed, but to the obstruction in the circulation as stated above. Authors are accustomed to speak of different degrees of retroflexion. Thus, the first degree is where the uterus has a slight bend backward, and the second is where it has a more marked flexion. 'The third degree is where the fundus is so much bent backward as to have the two extremities of a semicircle nearly touch 6 ach other. Sims used to state that the normal position of the unimpregnated uterus is on a line parallel with one from the umbilicus to the tip of the coccyx, this to be determined by the introduction of the uterine probe or sound. Any deviation from this position, which constitutes an angle less than forty-five degrees, is, as a rule, not to be considered as abnormal. The symptoms of uterine displacement are numerous. One of the most common is uterine dyskinesia, or painful and difficult locomotion. 'This symptom may occur with either variety of flexion, and become so aggravated as to acquire the title of reflex paraplegia. In such a case the power of locomotion is greatly diminished, and the action of the miscles is deficient. According to a more recent view by Dr. C. J. Nixon (Dublin Journal of Medical Science; Journal of THE AMERICAN Medical Association, vol. iii, p. 408), the dyskinesia or paraplegia is the result of an ascending neuri. tis or phlebitis which extends from the primary source of the disease of the uterus to the spinal cord, or the occurrence of a descending neuritis commencing in the sacral plexus, and extending downward along the sciatic nerves.

I have now under treatment a young lady unmarried, who has suffered greatly from uterine dyskinesia as a result of a retroposed uterus in the second degree, and whom I have greatly relieved by paying close attention to the uterine displacement. In her case the local application of tannin and glycerine continued for several weeks, coupled with good tonic constitutional treatment, has in a great measure relieved her distressing symptoms and enabled her to walk again as heretofore. 'The glycerine, according to its well-known action, abstracts fluid from the thickened and œdematous parts, while the tannin by its astringent power gives support to the vaginal and uterine walls. In another case I have used alum instead of tannin, in accordance with the plan of Robert Bell, M.D. (physician to the Glasgow Institution for Diseases of Women, Retrospect, part 89 , page $\mathbf{1} 65$. ) According to this author the tampon when saturated with a solution of alum in glycerine and applied in flexions or versions acts in three different ways; I. as a support; 2 . as a depleting agent; and 3 . as an invigorating agent to the uterus and vagina. That this agent by its styptic powers reduces the arterial supply, and by jts astringent property induces contraction of the muscular fibres, and thus materially assists in the expulsion of the venous blood. It has another advantage in being a cleanly application; it coagulates the catarrhal discharge and thus prevents its decomposition and the excoriation and vaginit is consequent on profuse and acrid discharges. Under this treatment papillary ulceration speedily disappears and hypertrophy of the cervical tissue greatly diminishes. I have recently had a case of a multipara, aged forty years, who had been a great sufferer from a retroposed uterus of the third degree. She had suffered much from hyperæsthesia extending to the left ovary, she had also suffered from cellulitis. The uterus could not easily be replaced, owing to firm adhesions. There was a good deal of unhealthy discharge, she had had an unfaithful husband. I tried a great variety of pessaries, but she experienced no benefit from their application. Indeed, it was with much difficulty that a pessary could be adjusted, as the uterus was held so firmly by the adhesions. This lady suffered much from cystitis, owing to the pressure of the cervix and body of uterus upon the bladder. As this woman had a very fair perinæum, I was advised to try tampons of glycerine and tannin. The applications were to be made every day or two, while the patient was in the recumbent position. These tampons were applied with gentle pressure at first, and each succeeding one was applied with more and more pressure, when at length the adhesions gradually gave way, and the uterus could be raised higher and higher toward the normal health line. 
The hyperasthesia and vaginitis finally disappeared, and the leucorrbœal and vaginal discharges ceased, and the mucous follicles about the cervix became atrophied. These medicated tampons were continued for some time after her more distressing symptoms had yielded.

In another case I used alum instead of tannin, as recommended, which is more cleanly, besides it gave equal if not better satisfaction. Following is the formula : glycerine, 320 grammes; alum, 40 grammes ; acid carbolic, if deemed necessary, 8 grammes; water, q. s. and a tampon of absorbent cotton saturated with the mixture is to be placed well up in Douglas's pouch, the other larger one behind the cervix and acts as support of the smaller of the two. This treatment is applicable to the bladder symptoms of anteversion or anteflexion, as a rule one tampon is sufficient (Retrospect, part 84, pages 20I-2). I recall another case of retroflexion which occurred in my practice about a year since, where a similar treatment was so far successful that the patient could dispense with the use of a pessary without inconvenience. Before she came under my care she had worn several kinds without material benefit. In another case where the retroversion was dependent upon a subinvolution the application of the medicated tampons, and small and repeated doses of ergotin, in pills three centigrammes, three times a day with gentle laxatives, effected a thorough and speedy recovery. In another case where I freshened the edges and closed an old ruptured perinæum for rectocele, 1 employed medicated tampons as a preparatory treatment. This case did well but a Hodge pessary was afterward necessary for a long time. In an old and troublesome case of prolapse, where there was a very fair perinæum, occurring in a Swedish lady, I succeeded quite well with the tampon but a good sized Hodge pessary afforded her great comfort.

Cases of anteflexion with or without cystocele are often quite troublesome and difficult to relieve. The following case came under my care: Miss H., unmarried, aged $x 6$ years, had suffered for some years more or less from an affection of the bladder. She suffered from symptoms simulating calculus of the bladder. Her suffering at length became so severe that medical attendance was imperative. This lady suffered from bradysuria, and the urine contained a copious sediment of mucous or white corpuscles. A thorough exploration of the bladder demonstrated that there was no calculus, but that the uterus was sharply anteflexed or bent upon itself like a joint of a finger when closed. The uterus, while the patient was under ether, was replaced by means of a uterine sound. Considerable pain and constitutional disturbance followed the operation, but this soon subsided. This patient at the time was seen in consultation by one of the most distinguished surgeons of Boston, who advised against the use of a pessary in this particular case, relying upon general treatment and occasionally restoring the displaced organ, but contrary to his advice a Hodge pessary was fitted and applied with seeming benefit and relief to the patient. This was by the advice of a specialist in this line of work.
This case I have had under observation and care for the past six years or more, and though the patient was undoubtedly relieved by the hot uterine douches at first continuously and systematically employed, yet I have had some doubts whether the pessary has been of that particular benefit which it at first seemed to promise. For I cannot forget what J. Matthews Duncan has said (Retrospect, part 87, page 206)-that a kindly doctor makes an amiable patient anxious to please him and ready to express a sense of relief which may not be real. Our patient was all this; a highly amiable lady, trained with great care, and anxious to please, and undoubtedly ready to express a sense of relief which may not have been real, without having a desire to be untrue. 'This young lady, contrary to the advice of Punch to the young man, not only contemplated marriage, but recently has become a wife to a sturdy Saxon. The writer of this paper has not lo:t his interest in the case, but still has his lenses properly focused, in order to see, not only what is to be the outcome of this case, but further, to ascertain who had the sounder opinion-the distinguished surgeon or the presuming specialist. One practice, however, that was at first offered, that of replacing the uterus from time to time by the probe or finger, does not receive the sanction of $\mathrm{J}$. Matthews Duncan. This, he says, has no uther effect than to irritate the organ, for the displacement immediately recurs after the probe or finger is removed. Juncan further, speaking of the use of pessaries, says he has never used one on any ground whatever in a virgin, and as to the benefit of pessaries for anteflexion, he expresses no belief, but remarks, if they do good, it is not by keeping the uterus straight; it is by diminishing or preventing further descent of that organ. He remarks he has never uied an anteversion pessary, and that he has no idea or intention of so doing, as he has never seen any benefit from their use. Matthews Duncan, in the same part (87, page 282), speaking of minor displacements of the uterus, says we may have these displacements without the symptoms, and the symptoms without the displacement, and until we know better than we know now that the symptoms are dependent on the displacement, he advises us to leave the displacements alone.

Now after reading carefully the above article, I cannot but feel that the author here referred to fails to distinguish a pathological condition of the uterus from a normal physiological one. According to the statistics of Dr. Vedeler (Archiev für Gynäkologie, retrospect, part 86, p. $19^{6}$ ) who examined 3 , ० 2 women, found 18 suffering from prolapsus. Of the remainder, in 15 per cent., the position of the uterus was normal, it was anteverted in 12 per cent., retroverted in ro per cent., anteflexed in 54 per cent. So that of 3,O I 2 women of the menstrual age, and of all conditions, the uterus was anteflexed in more than half of the number. Of the whole number 466 were virgins, 749 nulliparous, $\mathbf{3 2 2}$ were from 2 to 3 months pregnant, and $\mathrm{x}, 465$ were mothers. In healthy nulliparous women the normal position was found in 9 per cent. only, and anteflexion $7 \mathrm{r}$ per cent. In nulliparous women who complained of pelvic symptoms 
the normal position was $\mathrm{r} 5$ per cent. and anteflexed in $5^{6}$ per cent. In about 75 per cent. of healthy women who are nulliparous, the uterus was in a state of anteversion or anteflexion, while a similar position of the organ was found in 70 per cent. of such women who complained of uterine suffering, and the socalled normal position was found in 8 per cent. only of such women in health, but in 13 per cent. where they suffer from uterine disease. Emmet early recognized that versions may exist an indefinite period without causing disturbance, and it is only when the circulation is obstructed that discomforts arise. have a patient who has had a marked retroversion for the past eight years at least, and who has not had the slightest inconvenience from the displacement. In her case there is no impediment to the uterine circulation. I can also recall several cases of well-marked uterine displacement where no untoward symptoms have occurred. It is only in retroversion and in prolapse that mechanical means are of use. For this statement we have the authority of Emmet. As for anteversion ${ }^{1}$, that is for the most part the normal condition of the uterus, and it is only where we have prolapse or descent of the organ that vesical disturbances occur. The descent causes traction downward on the neck of the bladder or along the anterior wall of the vagina, just as retroversion causes the dragging of the neck of the bladder upward. Now in order to relieve the bladder in consequence of the anteflexion we must correct the prolapse of the uterus as we do in cases of vesical disturbance arising from retroversion. This was the condition in the case of my patient suffering from the effects of the anteflexion already referred to when a Hodge pessary was employed to elevate the uterus without regard to the flexion of that organ.

The same end is sought to be compassed by a new method of treating the severer uterine prolapsions by operation on the round ligaments (by William Alexander, M.D., F.R.C.S., England, Surgeon to the Liverpool Work-house Infirmary). Hence we see, from a therapeutic point of view, why, before the advance of gynæcology, the term prolapsus uteri was employed to embrace all that is comprised in the several terms that now denote uterine displacements. The operation devised by Alexander is not limited to cases of prolapsus uteri as such, but it has been resorted to with success in cases of retroversion, attended with descent of the uterus. Cases of cystocele, with prolapse of the vaginal wall, where there are no adhesions, may be slightly benefited by the operation, but where there are old adhesions this operation should not be undertaken. Drs. Campbell, Adams, Lediard and others have had recourse to this operation with success. Dr. Knowsley Thornton (Retrospect, part 82, p. 28r), speaking of uterine displacements, says it is the descent of the uterus and the dragging on the ovaries and broad ligaments, with consequent interference with the functions of the bladder and bowels, that lead patients to seek our aid. For extreme cases, where there is no descent,

\footnotetext{
1Since finishing this article I find just published in the Boston Medical and Surgical Journal, vol. cxi., p. 394 , October $23,188_{4}$, the fact alluded to by $\mathrm{Dr}$. Fifield that in foetuses and young infants the uterus is anteflexed to by Dr. Fifield that
}

but where mechanical obstruction exists in the cervical canal, he resorted to graduated suunds for their relief. This practice I have myself adopted, in several instances, for some years past, with quite good success. In such a case now, I make use of rapid dilatation as being more satisfactory. In regard to stem pessaries, Thornton believes them to be often of incalculable mischief. $\mathrm{He}$ believes those who take out pessaries, as a rule, do infinitely more good, and infinitely less harm than those who put them in. The writer of this article remembers once to have heard the late Dr. Atlee make the remark that he was often consulted in cases where he removed a pessary, but rarely in a case where he deemed one necessary. The advice contained in the above remarks, in regard to the use of pessaries, strikes every one as being somewhat sweeping, but perhaps was aimed at the practice of the cranks in the profession, who often can not discriminate between a case of simple retroversion and one requiring the aid of this valuable instrument. Besides the means above referred to, cases have been relieved by other modes of practice. Dr. Evory Kennedy (late Master of the Dublin Lying-in Hospital, Med.Press and Circular, Sept. 25, I $87_{2}$ ), makes mention of an obstinate case of procidentia cured by the actual cautery.

After the uterus was placed in situ the actual cautery was then applied about an inch and a half from the vulva round the surface of the vagina for the extent of about half an inch. The cicatrix formed by the eschar prevented the descent of the os. In my early practice, I remember treating a case of old and obstinate prolapsus uteri by industriously applying for several days, for the profuse leucorrhœal discharge, a strong solution of nitrate of silver to the protruding cervix. 'The uterus I had previously restored, but a violent inflammation ensued, that resulted in such firm adhesions I was never afterward able to distinguish clearly between the cervix and the fundus of that organ. Suffice it to say that the patient finally recovered from all her distressing symptoms, and regarded her case as having been most skillfully treated. In the same paper we find reported a case of procidentia vesicæ treated by a vaginal mould. A cast of the vagina was taken in wax, with the bladder restored to its natural position. A plaster of Paris mould was made from it, from this a caoutchouc instrument was moulded. This the patient wore with great comfort and convenience. Dr. James Dunlop reports a case of artificial occlusion of the vagina for the cure of prolapsus uteri. In this case, a strip of mucous membrane an inch and a half wide was dissected from half an inch below the meatus on one side to the corresponding point on the other. The raw surfaces were brought together, and kept together by means of quilled sutures. This patient was dismissed as having recovered. (Med. Times and $G a$ zette, Feb. 26, I 876), Dr. Protheroe Smith speaks of having used a pelvic band in the treatment of uterine versions. The main feature of his appliance is that it forms an artificial skeleton, and so supports all the chief points d'appui by which the complicated muscular systems engaged in the acts of expulsion harmoniz arid effect their purpose. As regards the choice 
of pessaries, Gaziel's air ball or air ring is often of benefit. I have mentioned having used Hodge's pessary; this I have found as good as any. Otto's spiral pessary I have often used, it is useful in cases of a temporary treatment. Graily Hewett's ring pessary is highly praised by many. It consists of a copper wire ring coated externally with gutta percha or soft rubber. The wire is of various diameters, and can be moulded into any shape the case may require. It is suitable for cases of anteversion, retroversion and prolapse. Thomas's anteversion and retroversion pessaries are often of benefit-I have used both with seeming advantage. From time to time many surgical operations of a heroic nature, such as the removal of the cervix, the entire uterus, the narrowing of the vagina, have been undertaken for the relief or cure of obstinate cases, but no operation is more satisfactory, in properly selected cases, for the relief of the distressing symptoms connected with uterine displacements, than the one undertaken for the restoration of a ruptured or deficient perinæum to its normal dimensions.

\section{MEDICAL PROGRESS.}

\section{MATERIA MEDICA AND THERAPEUTICS,}

Epilepsy and Bromism. Bromide of Potassium. - Prof. Germain Sée, in La Semaine Médical, dis. cusses this subject at considerable length. He considers first:

What is the part played by the potassium and the bromine in bromidle of potassium? Since the investigations of Traube the effect of the potassium salts, particularly of the nitrate, upon the heart has become recognized. The heart, after a short period of excitement, undergoes a certain degree of inhibition with diminution of the blood pressure; hence the conclusion that all the salts of potash constituted the true therapeutic method. But experience has shown that the chlorate, nitrate, and iodide of potassium are all perfectly inert in the treatment of epilepsy. It is therefore proven that potash in general, no matter in what dose it is given, does not possess the slightest therapeutic influence in epilepsy.

Another view of the action of bromide of potassium is to divide its physiological action into two parts, recognizing on the one hand its effects upon the brain, the spinal cord and the skin as of a special nature, and on the other, its effects upon the circulation, respiration and heat, as of a general nature and alkaline in its origin. The latest researches of Krosz show that in man the whole action is due to the bromine, while in animals the amount of bromine that can be taken as bromide of potassium without producing the slightest effect is enormous.

A third view not only removes all curative properties from bromide of potassium, but gives to it a baneful influence upon the heart, an intoxication of the heart. Here the effects of the bromism are attributed to the potassium, and another alkaline base is sought for, to replace the potassium, in order to avold the dangers that might result from its constant use for months and years.

It is this theory that has given us bromide of sodium, bromide of ammonium, and the combining of the two with the inevitable bromide of potassium. The poly-bromides, notwithstanding their complex effects, are now very much in vogue on account of this potassiophobia. The ordinary dose of 6 grammes of bromide of potassium introduces but 2 grammes of potash into the system. Under other circumstances the stomach has never made the slightest objection to this amount. The I I/2 grammes of potash to every 100 grammes of potato has never been suspected to possess a sedative action or injurious effect. To produce effects equivalent to 5 grammes of bromide of potassium, it is necessary to carry the dose of bromide of sodium to io or 15 grammes. So, to avoid a very uncertain danger, the risk is run of producing bromism. Injections of bromide of sodium into the blood of animals produces convulsions as with the potash salt. Bromide of ammonium is still more exciting, so that the three salts combined present no advantage over the bromide of potassium, which plays the third part in the polybromic mixture.

Absorption and dose. The bromine is readily and promptly absorbed by all the mucous membranes; in a few minutes it appears in the urine; it is true that it takes only two or three days to be thoroughly eliminated, therefore there is an imperious necessity for continuing its action without interruption; to omit a day occasionally or to diminish the dose is permissible, but to stop it altogether is dangerous. Professor Sée has seen patients where, the drug being stopped for several days in succession, convulsive attacks have recurred after an interval of a year or eighteen months. The bromine is eliminated in great part by the kidneys, very little by the salivary glands, still less by the stomach, and these organs are but little influenced by the drug. The respiratory mucous membrane and the skin, on the other hand, are deeply impressed by the elimination of bromine. The pharyngeal mucous membrane becomes the seat of a painful rasping sensation and of a very pronounced pallor, due to local ischæmiaand with a dose of 5 to 6 grammes the uvula becomes more or less completely anæsthetic. Voisin considers this a sign of saturation of the system-and as produced when Io or I 2 grammes have been taken, but individual characteristics in this respect differ so greatly that poisoning might occur before this symptom presents itself.

Woillez has tried to anæsthetize directly the palate by means of strong bromide gargles, but without success, - nor has touching the parts with the bromide itself proved efficacious. The bronchi become frequently the seat of marked irritation, from the passage of the bromine through the secretory glands; this bromic bronchitis results in a little irresistible cough, dry, or with but little expectoration, and is one of the most serious obstacles to the proper treatment of epilepsy. In one case the patient could never take more than one and a half grammes on 